\title{
Due vie per la semiotica o un incrocio di sguardi? Algirdas Greimas e Umberto Eco a confronto
}

\author{
Patrizia Violi \\ Università di Bologna, Italia \\ patrizia.violi@unibo.it
}

\section{Sommario}

Questo articolo intende confrontare criticamente il pensiero di due grandi semiotici scomparsi: Algirdas Greimas e Umberto Eco. Spesso contrapposti come caposcuola di due teorie semiotiche antagoniste - la semiotica generativa e quella interpretativa - ad uno sguardo più attento rivelano invece una comune base epistemologica. In particolare è l'assunto strutturalista di fondo ciò che costituisce l'orizzonte condiviso delle loro semiotiche, da cui discendono posizioni simili su molti altri punti, dall'anti essenzialsimo alla narratività. Su altri aspetti invece, come l'enunciazione e il metalinguaggio, gli approcci divergono, pur all'interno di una stessa indagine sul senso e i linguaggi che al fondo li accomuna.

Parole chiave: Greimas, Eco, teoria semiotica, senso, metalinguaggio.

\section{Two ways for semiotics or a crossing of looks? Algirdas Greimas and Umberto Eco in comparison}

\begin{abstract}
This article aims to critically compare the thought of two great disappeared semiotics: Algirdas Greimas and Umberto Eco. Often opposed as the chief of two semiotic antagonistic theories - generative and interpretative semiotics - a more careful look reveals instead a common epistemological basis. In particular, it is the underlying structuralist assumption which constitutes the shared horizon of their semiotics, from which similar positions arise on many other points, from the essence to narrative. On the other hand, such as enunciation and metallic language, the approaches diverge, even within a same survey of the meaning and the languages at the bottom of them.
\end{abstract}

Keywords: Greimas, Eco, Semiotic theory, sense, metalanguage. 


\section{Tempo di bilanci?}

Il 2017 è stato l'anno del centenario della nascita di Algirdas Greimas e a lui sono stati dedicati numerosi convegni in ogni parte del mondo. Un anno prima, il 19 febbraio 2016, moriva Umberto Eco, lasciando scritto nel proprio testamento di non voler alcun convegno su di sé per almeno 10 anni dal momento della sua morte. Non ci saranno quindi, almeno per ora, momenti di approfondimento delle sue numerose e transdisciplinari ricerche ed è certo ancora troppo presto per qualsivoglia, seppure temporaneo, bilancio critico.

La concomitanza temporale di questi due eventi ci induce tuttavia a ripensare al lavoro di quelle che indubbiamente sono state le due più importanti figure della semiotica contemporanea. Dopo la generazione dei padri fondatori, Greimas e Eco costituiscono senza alcun dubbio i punti di riferimento imprescindibili della nostra ricerca, nonché gli studiosi con cui almeno i più anziani fra noi si sono formati.

Quale relazione, quali punti di contatto, quali differenze esistono fra di loro? La vulgata, ripresa per altro in quasi tutti $\mathrm{i}$ manuali a mia conoscenza, contrappone spesso come alternative e incompatibili due scuole semiotiche: la semiotica generativa, che ha in Greimas il suo fondatore ed è strutturalista nelle sue origini, e la semiotica interpretativa di Umberto Eco, di ascendenza invece peirciana.

Essendo sono anche io responsabile in prima persona di una lettura che, se non propriamente falsa è però certamente riduttiva, mi sento oggi impegnata a rileggerne $\mathrm{i}$ termini in un'ottica più approfondita.

La mia responsabilità va fatta risalire al lontano 1982, anno di pubblicazione di un numero monografico di VS. Quaderni di studi semiotici, la rivista fondata e diretta da Umberto Eco, dedicato alla analisi di un testo, Sylvie di Gerard de Nerval. Umberto Eco aveva proposto ai suoi studenti e collaboratori all'Università di Bologna un seminario collettivo su quel testo, a cui poi molti studenti dedicarono la loro tesi di laurea.
Sylvie è un testo particolare, caratterizzato da quello che e' stato definito "effetto nebbia", per la difficoltà che suscita nel lettore nel definire l'esatta sequenza temporale degli eventi e perfino il loro statuto di esistenza: presente e passato, ricordi e sogni, realtà e fantasia si alternano e sfumano l'uno nell'altra, senza che sia spesso possibile determinare con certezza in quale universo testuale ci troviamo. Il testo quindi si prestava molto bene sia a ricostruirne il complesso percorso narrativo e le trasformazioni che il narratore subiva nel corso del tempo, sia gli smarrimenti interpretativi del lettore.

Alla fine del seminario facemmo una pubblicazione, il numero 31-32 di VS, a cui Eco mi chiese di fare l'introduzione (Violi 1982). Per dare conto di questi due sguardi diversi, io utilizzai allora le due denominazione di semiotica generativa e interpretativa, una distinzione che ebbe poi un enorme successo. Un successo in parte immeritato, nel suo semplificare e contrapporre troppo semplicisticamente una situazione molto più articolata.

$E^{\prime}$ ora tempo di ripensare criticamente quella distinzione per mettere in luce, insieme alle differenze, anche $\mathrm{l}^{\prime}$ importante matrice comune che soggiace alle due semiotiche. Solo in questo modo saremo in grado di comprendere appieno la posta in gioco di un progetto epistemologico che ha radici profondamente condivise, un progetto che, iniziato nel secolo scorso, è al tempo stesso teorico e analitico.

Questo non significa forzare omologie indebite, ma capire meglio punti di incontro e di distanza, snodi possibili. Non definitivi perché, come cercherò di mostrare alla fine del mio intervento, gli sviluppi più recenti delle cosiddette due semiotiche presentano oggi punti di intersezione nuovi, particolarmente interessanti e produttivi.

Partirò dunque dallo smontaggio critico di alcunefalseopposizionicheattraversanoildiscorso semiotico, iniziando dalla contrapposizione più diffusa, quella fra una semiotica strutturalista e una interpretativa peirciana, lette spesso come tradizioni antitetiche. 


\section{Strutturale vs interpretativo}

Va ribadito subito con fermezza che le cose non stanno affatto così: tutta la tradizione semiotica ha una matrice strutturale, sia in ambito linguistico che filosofico che antropologico. L'idea più costitutiva dello strutturalismo è senza alcun dubbio quella di differenza: i singoli elementi non hanno un valore in sé, assoluto, ontologico, essenzialmente definito una volta per tutte, ma acquisiscano valore e senso solo dal confronto con ciò che non sono, dunque solo nel valore posizionale della relazione con altro da sé.

E' quindi la relazione differenziale a determinare il senso, a specificare il significato di un dato termine nel sistema linguistico, come insegnava Saussure, o a determinare la funzione e il ruolo narrativo che un dato attore ricopre in un racconto, come tutta la tradizione narratologica strutturale ci ha insegnato, da Propp fino alla teoria narrativa di Greimas.

Ora questa idea non è affatto contrapposta alla tradizione filosofica peirciana a cui si ispira la semiotica interpretativa di Eco. Vi è un importante saggio di Peirce (1897: CP 3.456-482) che si intitola La logica dei relativi, dove Peirce sviluppa precisamente l'intuizione che $\mathrm{e}^{\prime}$ alla base dello strutturalismo europeo, e cioè la priorità della relazione sui singoli termini. Non a caso Jakobson ha sostenuto che Peirce era il primo degli strutturalisti.

D'altra parte Eco, fin dal Trattato di Semiotica del 1975, nella sua critica ai modelli semantici dizionariali, propone un'idea relazionale e rizomatica del senso, dove ogni nodo acquista il suo senso solo in relazione ad altri nodi, che non sono mai pre-definiti e dati, ma dipendono sempre dai percorsi enciclopedici che il processo interpretativo induce.

Si potrebbe osservare che Greimas non parla mai di Enciclopedia né prevede alcun concetto paragonabile ed indubbiamente questa è una differenza rilevante. Il mio intento tuttavia non è certo quello di sostenere una sovrapponibilità o omogeneità totale fra i due modelli, ma piuttosto di decostruire alcuni luoghi comuni che si sono venuti sviluppando all'interno della koinè semiotica, stratificando interpretazioni tanto stereotipate quanto superficiali.

A questo fine mi pare urgente evidenziare alcuni comuni parametri epistemologici imprescindibili, di cui il primo è senz'altro la comune matrice strutturalista che, alla base di entrambe le cosiddette due semiotiche, ne costituisce una delle radici più profonde, pur nelle differenti declinazioni reciproche.

Eco legge la relazionalità sullo sfondo, illimitato, di una enciclopedia che contiene potenzialmente tutto il sapere umano e le cui vie sono percorribili in infiniti modi, Greimas è più interessato alle dipendenze che si vengono ad instaurare all'interno di ogni singolo testo. Pur nella differenza di prospettiva e di sguardo, comune e condiviso è l'orizzonte relazionale e differenziale da cui si sviluppa il senso.

\section{Anti essenzialismo}

Dall'opzione strutturalista di fondo discendono alcune conseguenze epistemologiche di grande rilievo in entrambi gli approcci. In primo luogo quella che possiamo definire come una posizione profondamente anti essenzialista: se la relazione vien prima del singolo elemento non ci può essere nessuna essenza intrinseca a cui ancorare la definizione dei singoli termini, in altri termini nessun ontologia soggiacente può farsi garante dei processi di costruzione del senso. Anche in questo caso un assunto di base comune viene raggiunto attraverso percorsi parzialmente differenti. fin dai tempi de $L a$ struttura assente, Eco prende le distanze da ogni deriva ontologica della struttura in favore di una lettura metodologica non essenzialista.

In Greimas non troviamo testi interamente dedicati alla discussione del concetto di struttura da un punto di vista teorico generale; anzi alla voce 'Struttura' nel Dizionario del 1979 (Greimas e Courtés 1979) viene esplicitamente detto che non si vuole "entrare nelle controversie filosofiche e ideologiche che la nozione continua a provocare". Greimas, più interessato alla costruzione di un sistema coerente ed 
esaustivo, preferisce non affrontare in modo diretto il dibattito filosofico. A mio parere tuttavia l'insistenza di Greimas sulla testualità, l'invito a partire sempre e soltanto da un testo, definendone i confini e analizzandone i livelli di senso, risponde ad una esigenza non dissimile da quella che Eco si poneva ai tempi de $\mathrm{La}$ struttura assente, e anche qui mi pare di scorgere una comune preoccupazione epistemologica.

Anche per Greimas non esistono strutture in sé, ma solo testualmente date. La struttura non è mai una Ur costruzione, ma qualcosa da rintracciare nei testi, da ricostruire e "riportare alla luce" in ogni singola analisi. E' questa la necessità posta con forza nel più volte ribadito principio di immanenza, così importante nella teoria greimasiana.

Se per Greimas il senso si dà sempre all'interno di una testualità data, anche nel lavoro di Eco si ritrova un' analoga ispirazione, se pur declinata un poco diversamente. Non dimentichiamo che Eco ha iniziato la sua carriera proprio con l'analisi di testi, sia appartenenti alla cosiddetta cultura alta che a quella popolare e di massa, rivendicando un principio e una metodologia profondamente 'immanentista', anche se Eco non usa questa parola. L'intento di Eco infatti non era infatti certo quello di omologare la Divina Commedia a Topolino, come gli è stato spesso rinfacciato da critici poco avveduti, ma al contrario di indagare testualità diverse con criteri metodologici coerenti e trasversali. Quello che a Eco stava a cuore era mostrare come il criterio di 'qualità' di un testo non passasse per classificazioni esterne, di tipo socioculturale, come quella di genere implicita nella dicotomia "cultura alta" vs "cultura popolare", ma fosse da ricercare proprio in un rigoroso lavoro sulla testualità. La metodologia semiotica era per Eco lo strumento principe per arrivare a una maggiore intelligibilità dei testi, anche quelli più popolari e di consumo, secondo uno spirito non diverso da quello con cui Greimas analizzava la soupe au pistou.

Come ho appena detto, Eco non usa mai il termine immanenza. Quando ne ha teorizzato, Eco lo ha fatto con un linguaggio differente, più vicino a quello dell'epoca in cui scriveva. Questa è una interessante differenza fra i nostri due semiologi: Eco si è sempre molto confrontato con il dibattito delle discipline limitrofe, formulando le sue ipotesi di volta in volta all'interno delle discussioni in corso anche al di fuori del circolo semiotico. Discutendo, in Semiotica e Filosofia del linguaggio, della distinzione fra semantica e pragmatica, Eco ribadisce l'impossibilità di una semantica che non sia al tempo stesso una pragmatica e che quindi non si faccia carico di un preciso contesto di interpretazione. Per Eco "le regole per la generazione e l'interpretazione delle espressioni di un linguaggio sono sempre ancorate a uno specifico contesto" (Eco 1984: 69).

Come si vede, la formulazione è diversa: Greimas parla di testo, e non usa mai come riferimento il dibattito del tempo su semantica e pragmatica. Eco invece ha sempre, nelle sue argomentazioni, uno sguardo allo 'spirito del tempo' e ai dibattiti che stanno avendo luogo in ambito linguistico e filosofico. Credo tuttavia di non forzare l'interpretazione dei testi mettendo in parallelo la necessità di Greimas di circoscrivere il senso sempre all'interno di una forma testuale e quella echiana di un'interpretazione che è sempre localmente guidata e delimitata dal suo specifico contesto, dal suo intorno.

\section{Testo aperto vs testo chiuso.}

Un altro equivoco in cui ci si imbatte frequentemente riguarda la questione dell'apertura o chiusura dell'interpretazione testuale, spesso viste come posizioni contrapposte all'interno delle due semiotiche. Secondo una simile lettura, Eco sarebbe per l'apertura interpretativa, Greimas per una rigorosa definizione dei confini testuali. Forse l'equivoco si può far risalire al libro di Eco del 1962 Opera aperta, ma si tratta anche in questo caso di un luogo comune da sfatare. Nella teoria di Eco la apertura interpretativa è strettamente delimitata e le letture possibili sono tutt'altro che 'aperte', tanto che ai tempi dell'uscita di Lector in fabula, e poi del successivo I limiti dell'interpretazione, Eco venne molto criticato proprio per questa sua chiusura, che veniva, indebitamente in realtà, contrapposta alla presunta apertura di Opera Aperta. 
Le critiche arrivavano da posizioni più vicine al decostruzionismo, che rivendicava una quasi totale libertà di letture. Le posizioni di Eco su questo punto hanno un po' oscillato proprio in relazione alla posizione prevalente nel dibattito a lui contemporaneo. Eco scrive Opera aperta e $\mathrm{La}$ Strutturaassentenelmomento di maggior influenza dello strutturalismo, quasi a controbilanciarne il rigore, e poi il Lector e i Limiti quando invece pare dominante, sulla scena del dibattito internazionale, la posizione del decostruttivismo, a cui Eco si oppone in maniera netta e radicale. Anche qui possiamo ritrovare una differenza fra i due autori: Eco più volto a dialogare con i contemporanei, Greimas più attento a elaborare il suo proprio sistema, cosa che Eco in fondo non farà mai: non esiste infatti un "modello" o schema echiano per l'analisi dei testi.

Da questo punto di vista il sistema greimasiano appare più "compatto" e coerente, nel senso che non ci sono spostamenti significativi sulla questione del rapporto tra testo e interpretazione. Anche negli ultimi lavori sulle passioni e gli effetti estesici, l'attenzione è ancora al testo e alle sue dinamiche immanenti. Esemplare in questo approccio è proprio il suo ultimo testo (De l'imperfection) quasi un testamento.

Greimas non si pone mai in maniera esplicita il problema dell'interpretazione, che consiste fondamentalmente nel ripercorrere a ritroso quello che è il percorso generativo di un testo, ricostruendone il senso nella sua strutturazione per livelli secondo per l'appunto il principio di immanenza testuale.

A ben vedere tuttavia anche il Lettore Modello di Eco, concetto guida della interpretazione testuale, è una dimensione strutturalmente immanente al testo, essendo null'altro che la strategia interpretativa che l'autore ha iscritto nel testo stesso per guidarne e orientarne la lettura. In Eco certamente questa strategia non ha la forma della ricostruzione a ritroso, in profondità, per livelli testuali successivi. Tuttavia questo dato mi pare meno rilevante della natura interamente testualista delle procedure di interpretazione in Eco, che ha sempre combattuto ogni forma di apertura decostruzionista. Da questo punto di vista il testo, per Eco, è chiuso quanto lo è per Greimas, e la sua chiusura è iscritta precisamente nella struttura del testo stesso. Si pensi, per chiarire meglio questo specifico punto, alla distinzione che, ne I limiti dell'interpretazione, Eco propone fra 3 tipi di intentio, la intentio autoris, la intentio operis e la intentio lectoris. Solo la intentio operis è quella che conta ai fini interpretativi, ma l'intentio operis altro non è che la struttura interna iscritta nell'opera, indipendentemente dalle intenzioni dell'autore a significare, e da quelle del lettore a interpretare. Solo ciò che è nell' opera e nella sua intentio è interpretazione legittima, il resto è "uso" secondo la nota distinzione echiana di uso e interpretazione.

Caso mai a Eco interessa un aspetto che per Greimas è del tutto irrilevante, e cioè come nel testo siano disseminati indizi che portano il lettore a quel dato percorso interpretativo che è quello previsto dal testo stesso. Quindi non la ricostruzione, a ritroso, partendo dalla manifestazione per risalire all'immanenza, dei percorsi per livelli che strutturano il testo, come in Greimas, ma piuttosto i "salti" le anticipazioni, le scommesse di lettura, e le eventuali "sorprese" iscritte nel testo dall'autore. Significativi in questo senso sono i tipi di testi "esemplari" su cui si esercitano le letture dei due semiotici: da un lato un testo che potremmo definire classico come il Maupassant dei Deux amis, dall'altro testi che sono quasi delle macchine per ingannare il lettore come il Drame bien parisien di Alphonse Allais o Silyie con il suo effetto nebbia della, due testi che iscrivono entrambi al loro interno uno spiazzamento di lettura, seppure con modalità ed esiti differenti.

Nella predilezione di Eco per testi fatti apposta per sorprendere e spiazzare il lettore nel suo sforzo interpretativo, nel suo corpo a corpo con il testo, si può certamente rintracciare la sua passione per il ragionamento abduttivo, certo ereditata da Peirce, e in ultima istanza per il suo interesse a una teoria della conoscenza generale, prima ancora che a una teoria del testo.

Inaltri termini, e semplificando, là doveGreimas si chiede: come è costruito il senso di questo testo, Eco si interroga su come arriviamo a capire il senso 
di quel testo, attraverso quali procedure di senso iscritte nel testo. La sua naturalmente non è una domanda di tipo psicologico ma epistemologico, sulle forme della conoscenza: come arriviamo a conoscere ciò che conosciamo?

Ma questa differenza di prospettiva, pur rilevante ad un altro livello, non riguarda l'apertura interpretativa del testo, su cui le posizioni dei due autori sono assai vicine.

\section{Narratività}

Un altro punto di affinità lo individuerei nel ruolo che la narratività ha in entrambi gli autori. Per Greimas come noto senso e narratività coincidono, la narratività è la forma del senso, il modo in cui il senso si dispiega, "il principio stesso dell'organizzazione di ogni discorso narrativo e non narrativo' (Greimas e Courtés 1979: 248).

L'idea che il senso si dà a noi solo nella forma della narratività è forse una delle più geniale scoperte teoriche di Greimas, alla base anche di tutti gli sviluppi odierni della semiotica post-greimasiana, da Landowski a Fontanille, che non si discostano mai da questo principio fondativo, rifinendolo, complessificandolo, arricchendolo di nuove prospettiva, ma mai prendendone le distanze.

Anche in questo caso io credo che pure Eco veda nella narratività la forma di base del senso, ma ci arrivi per altra strada. Già dagli anni 80, lavorando sui rapporti fra teoria semantica e teoria del testo Eco parlava del semema come testo virtuale, cioè come un micro-testo che condensava un'intera storia, seguendo in questo il principio di condensazione e espansione che regola il funzionamento linguistico ricavato da Hjelmslev. Il semema "pescatore", per utilizzare un esempio di Eco stesso, racchiude un'intera storia, evoca un frame narrativo in cui è facile rintracciare ruoli attanziali e tematici, livelli figurativi, sistemi valoriali e passionali. ( $E^{\prime}$ mia convinzione che Eco abbia preso il termine "pescatore" non a caso, ma come un implicito omaggio al Maupassant di Greimas).
Ma l'idea di semema come testo virtuale serve a Eco non tanto per articolare una teoria del testo, quanto, di nuovo, per articolare una teoria della conoscenza. Il testo virtuale racchiuso nel semema ci permette di capire il testo perché è uno schema che ci spiega il mondo, ci fa capire il mondo in cui viviamo, le sue forme, le sue abitudini, i suoi abiti, per dirla con Peirce. Per questo gli schemi narrativi condensati nella lingua rimandano sempre ad una dimensione culturale, sono una sorta di precipitato enciclopedico potremmo dire, che ricorda quanto in tempi più recenti nella semiotica greimasiana è stato sviluppato nell'idea di prassi enunciativa o impersonale, proprio per spiegare come gli usi linguistici, legati e dipendenti dalle forme culturali, si depositino nelle nostre enunciazioni singolari.

Inquestosensorifletteresullaideadinarratività presente nel pensiero di Eco ci permette di capire ancora meglio quella differenza di prospettiva fra i due autori a cui accennavo prima. Infatti anche su questo punto conclusioni vicine e compatibili sono raggiunte attraverso percorsi, $\mathrm{o}$ forse preoccupazioni teoriche, diverse.

In Greimas la narratività è alla base del senso in quanto il senso si può solo cogliere solo per trasformazioni, in particolare trasformazioni dei soggetti e trasformazioni dei valori che essi incarnano. La narratività diviene in questo modo una teoria della azione umana, delle sue motivazioni - e quindi delle sue passioni - e dei suoi concatenamenti sintagmatici. Una teoria dell'azione e della passione.

Per Eco il problema torna sempre ad essere quello della conoscenza, di come conosciamo il mondo. E per Eco il mondo lo conosciamo sempre attraverso una possibile ipotesi che altro non è se non uno schema narrativo. Esemplare è a questo proposito la storia di Zadig analizzata da Eco: se Zadig non avesse costruito un racconto non avrebbe capito nulla degli indizi sparsi che gli si presentavano in modo apparentemente casuale $^{1}$ Il ragionamento abduttivo, perno della teoria della conoscenza che Eco deriva da Perice, è al suo fondo un ragionamento basato sulla

1 Cfr su questo C.Paolucci, Umberto Eco, Milano: Feltrinelli, 2017. 
narratività: costruire una storia plausibile che renda conto degli indizi che abbiamo e li renda coerenti all'interno di una narrazione. In una risposta ad un volume di scritti a lui dedicati del 2004 Eco lo dice in forma esplicita: “Dobbiamo interpretare il mondo come se fosse una storia o un testo" ${ }^{\prime 2}$.

\section{Divergenze}

\subsection{L'enunciazione}

Se finora abbiamo soprattutto evidenziato i punti di comunanza e convergenza all'interno delle due prospettive, è ora giunto il momento di riflettere anche sulle più importanti differenze delle due teorie, che investono soprattutto le nozioni di enunciazione e metalinguaggio. Iniziamo dalla prima delle due.

Come è noto l'enunciazione è il dispositivo che permette la mediazione tra il sistema della lingua e la sua esecuzione; la sua elaborazione è centrale nella teoria strutturale, a partire dalla formulazione di Emile Benveniste fino allo sviluppo successivo nella semiotica di Greimas.

Per questo autore l'enunciazione è riconducibile alle tracce lasciate nell'enunciato dall'atto stesso dell'enunciazione, che è alla base della produzione discorsiva ma è in sé inaccessibile. Attraverso un atto originario di "schizia creatrice" il soggetto dell'enunciazione si proietta, debrayandosi, nell'enunciato e vi iscrive le categorie della persona, dello spazio e del tempo (Io, qui, ora e non-io, non qui non ora).

Unquadro molto diversoè invece rintracciabile nella teoria echiana. Eco è stato spesso accusato di non avere una propria teoria dell'enunciazione, anzi di non averne alcuna, e questo è senz'altro vero se consideriamo l'enunciazione nel senso benvenistiano (e greimasiano), come un "Io che dice Io" lasciando le tracce della sua presenza nel testo enunciato. Ma l'assenza di una teoria dell'enunciazione discende direttamente dal modo in cui Eco pensa il soggetto e la soggettività nella semiosi. Per Eco non esiste un soggetto "forte" che prende la parola; al contrario ogni parola, ogni enunciazione, è già iscritta in una rete di altre parole, di altre enunciazioni che la precedono e la condizionano. La soggettività, in questo quadro, non è certo riportabile ad un soggetto dell'enunciazione di tipo husserliano, come nella linea Benveniste-Greimas, ma è piuttosto una soggettività diffusa, circolante, che di fatto viene a coincidere con le pratiche stesse della semiosi, o per usare i termini di Eco, "con le bave e i detriti" dell'Enciclopedia. La stessa semantica delle espressioni usate dai due autori è significativa: da un lato l'atto grandioso di una "schizia creatrice", dall'altro la più umile e residuale "bava e detriti".

Due visioni certo molto diverse che però, nei successivi sviluppi della teoria greimasiana, tendono ad avvicinarsi e a diventare più compatibili. L'idea di prassi enunciativa, elaborata da Denis Bertrand, Jacques Fontanille e Claude Zilberberg, si avvicina molto alla posizione di Eco, nell'attenzione posta al carattere impersonale (e non soggettivo) dell'atto enunciativo, e al suo fare sempre costante riferimento a un insieme di repertori, stereotipi e grandezze semiotiche comuni e soggiacenti, depositati nella cultura condivisa.

\subsection{Il metalinguaggio}

Infine vi è un ultimo aspetto di reale, e probabilmente inconciliabile, differenza, ed è la questione del metalinguaggio.

La costruzione di un metalinguaggio adeguato e rigoroso è alla base della teoria greimasiana, un metalinguaggio che deve essere al tempo stesso interdipendente, chiuso e specifico.

Il metalinguaggio ha in questo modello la precisa funzione di fornire interdefinizioni dei termini in modo preciso e univoco, all'interno

2 "A Response by Eco", in Ross, C. and Sibley, R., Illuminating Eco. On the Boundaries of Interpretation, Ashgate Publishing Company, Burlington, USA, 2004: 193-199. 
di un sistema chiuso, tale da non generare equivoci e da escludere altri usi, quali quelli del linguaggio comune ma anche della tradizione filosofica precedente.

Limitare la polisemia del linguaggio ordinario ha una sua precisa ragion d'essere teorica, quella di garantire una oggettività della analisi, insieme ad una possibilità di confronto intersoggettivo. Per Greimas il metalinguaggio serve a fondare la vocazione scientifica della disciplina; creare un linguaggio condiviso da una comunità scientifica che si identifica come tale proprio per l'utilizzo degli stessi strumenti teorici e metodologici diviene così una parte essenziale del suo progetto teorico.

Eco invece non crede alla possibilità del metalinguaggio, di livello distinto dal linguaggio oggetto, perché, peircianamente, ogni segno è ritraducibile in un altro nella catena degli interpretanti, quindi non si possono dare livelli di tipo metalinguistico.

Questo assunto teorico ha un'importante ricaduta, perché esclude di principio la possibilità di qualsivoglia interdefinizione che circoscriva il linguaggio analitico di una comunità e lo chiuda, precludendo proprio quella catena di ritraduzione che per Eco è alla base della semiosi. Al contrario, per Eco è importante proprio la strada opposta: risalire a tutte le accezioni di ogni termine teorico. Da qui la sua costante attenzione alla storia della semiotica e del suo pensiero, che nasce dalla necessità, per Eco, di farsi carico proprio di tutte le possibili accezioni e percorsi di senso stratificati senza escluderne nessuno, ma attraversandoli secondo percorsi interpretativi diversi. Un'idea rizomatica del senso e dei linguaggi che è alla base della nozione di Enciclopedia.

In questo la posizione di Eco è decisamente opposta a quella di Greimas, e forse non è un caso che Eco non abbia mai avuto, né cercato peraltro, una vera e propria "scuola" come invece è stato il caso per Greimas.

Più che un vero metalinguaggio comune, nel caso di Eco, esiste un "orizzonte di senso condiviso", punti di riferimento sempre variamente rileggibili e interpretabili. Come garantirsi un controllo intersoggettivo sulle interpretazioni in una simile situazione? Credo che Eco risponderebbe che il controllo non è tanto affidato alla comunità degli studiosi, via il metalinguaggio, ma piuttosto alla comunità generale degli interpreti, seguendo una intuizione di Peirce. In altre parole ciò che in Greimas è fissato a monte, con la condivisione di un metalinguaggio, in Eco è fissato a valle, dal controllo intersoggettivo della comunità.

Due strade diverse per garantire però un fine cheforsenonè poicosìlontano, perchéinentrambi i casi guarda al controllo, intersoggettivamente fondato, che una comunità può e deve esercitare sulle pratiche di analisi e interpretazione, anche se diversa è forse l'accezione di comunità a cui si guarda.

\section{Conclusioni}

Per concludere, ci si potrebbe chiedere, al di là di possibili convergenze o al contrario diversità, qual era, per questi due grandi semiotici, il senso più profondo della loro ricerca.

Sappiamo che per Greimas questo si riassumeva in una formula tanto semplice quanto incisiva: dire qualcosa di sensato sul senso. Tutta la sua teoria è una grande macchina per dare conto delle articolazione del senso, dalle strutture narrative profonde all'allestimento figurativo del mondo, ai moti passionali che lo attraversano, alle estesie che ne traspaiono.

Eco non si sarebbe espresso in questi termini. La sua matrice era più filosofica che linguistica o antropologica, e per lui la semiotica era la parte più essenziale della filosofia. Ma la filosofia per Eco era in primo luogo lo studio dei segni e dei linguaggi, nella convinzione che per arrivare a capire il mondo sia necessario in primo luogo capire come lo interpretiamo attraverso il linguaggio e i segni di cui ci serviamo, costante mediazione che si frappone fra noi e il mondo.

Se per Greimas lo scopo della teoria semiotica era arrivare a meglio spiegare il funzionamento 
del senso, per Eco la funzione della filosofia, per lui coincidente con la semiotica, era quella di accrescere la consapevolezza del funzionamento dei segni e dei linguaggi.

Al di là delle diverse, e anche importanti, divergenze che distinguono queste due direzioni della ricerca semiotica, credo che su questo punto questi due grandi che ormai ci stanno alle spalle sarebbero stati d'accordo.

\section{Referencias bibliográficas}

Eco, U. (1962). Opera aperta, Milano: Bompiani. (1968). La struttura assente, Milano: Bompiani.

(1975). Trattato di Semiotica, Milano: Bompiani.

(1979a). Semiotica e Filosofia del linguaggio, Milano: Bompiani.

(1979b). Lector in fabula, Milano: Bompiani.

(1990). I limiti dell'interpretazione, Milano: Bompiani.
Greimas, A. (1970). Du sens, Paris: Seuil.

(1976). Maupassant, la sémiotique du teste. Exercices pratiques, Paris: Seuil.

(1983). Du sens II, Paris: Seuil.

(1987). De l'imperfection, Paris: Pierre Fanlac.

Greimas, A. J. e Courtés, J. (1979). Sémiotique. Dictionnaire raisonné de la théorie du langage, Paris: Hachette.

Paolucci, C. (2017). Umberto Eco, Milano: Feltrinelli.

Peirce, C.S., (1931-58). Collected Papers (C.P.), Cambridge Mass: Harvard University Press.

Ross, C. and Sibley, R. (2004). Illuminating Eco. On the Boundaries of Interpretation, Burlington, USA: Ashgate Publishing Company.

Violi, P. (1982). "Du coté du lecteur", VS. Quaderni di studi semiotici, 31-32. 